\section{Kidney \\ Blood Pressure Research}

\title{
A High Fat Diet During Pregnancy and Lactation Induces Cardiac and Renal Abnormalities in GLUT4 +/- Male Mice
}

\author{
Michael Kruse ${ }^{a}$ Ariana Fiallo $^{a}$ Jianling Tao ${ }^{d}$ Katalin Susztak $^{d} \quad K^{2}$ erstin Amann ${ }^{e}$ \\ Ellen B. Katz ${ }^{a} \quad$ Maureen J. Charron ${ }^{a, b, c}$ \\ Departments of aBiochemistry; ${ }^{b}$ Medicine; 'Obstetrics and Gynecology and Women's Health, Albert \\ Einstein College of Medicine, Bronx, NY; ${ }^{d}$ Renal, Electrolyte and Hypertension Division, Department \\ of Medicine, University of Pennsylvania, Philadelphia, PA, USA; 'Nephropathology, Department of \\ Pathology, University of Erlangen-Nürnberg, Erlangen, Germany
}

\section{Key Words}

Cardiorenal Syndrome $•$ Fetal Programming $\bullet$ GLUT4 $•$ High Fat Diet $•$ Hypertension

\begin{abstract}
Background/Aims: Altered nutrients during the in utero (IU) and/or lactation (L) period predispose offspring to cardio-renal diseases in adulthood. This study investigates the effect of a high fat diet (HFD) fed to female mice during IU/L on gene expression patterns associated with heart and kidney failure and hypertension in male offspring. Methods: Female wild type (WT) mice were fed either a HFD or control chow (C) prior to mating with males with a genetic heterozygous deletion of GLUT4 (G4+/-, a model of peripheral insulin resistance and hypertension) and throughout IU/L. After weaning male offspring were placed on a standard rodent chow until 24 weeks of age. Results: All offspring exposed to a maternal HFD showed increased heart and kidney weight and reduced cardiac insulin responsiveness. G4+/- offspring on a HFD displayed early hypertension associated with increased renal gene expression of renin and the $A T_{1}$ - receptors compared to $\mathrm{G} 4+/$ - on a $\mathrm{C}$ diet. This group showed decreased cardiac expression of key genes involved in fatty acid oxidation compared to WT on a C diet. Conclusions: These results indicate an interaction between a HFD diet and genotype during early life development that can enhance susceptibility to cardio-renal diseases later in life.
\end{abstract}

\section{Introduction}

The cardiorenal syndrome (CRS) is a serious clinical condition of simultaneous heart and kidney failure. It is characterized by a complex pathophysiological interaction between the heart and the kidney in which one organ can be negatively influenced by the other, 


\section{Kidney Blood Pressure Research}

thus increasing overall morbidity and mortality [1]. Human CRS consists of five subtypes depending on the leading failing organ and the duration of the disease [2].CRS is a combination of clinical features including global heart failure, hypertension, reduced nephron numbers and hyperfiltration of the kidney [3]. Numerous studies have been performed to investigate the origin of CRS, however the pathophysiologic mechanisms have not been fully revealed. Oxidative stress along with other metabolic factors appears to play a role in promoting the onset of CRS [4].

Recently the term cardiorenal metabolic syndrome (CRMS) was established to acknowledge the increased prevalence of the metabolic syndrome coinciding with CRS [5]. The metabolic syndrome is characterized by obesity, insulin resistance and hypertension that can lead to cardiovascular diseases (CVD) including heart failure [6]. However, either hypertension or obesity alone can be associated with cardiac hypertrophy [7]. Specifically the prevalence of obesity in children has increased over recent years. These patients are at risk of developing cardiovascular and/or renal diseases due to insulin resistance, increased oxidative stress and inflammation as a result of long standing obesity.

Oxidative stress in the failing heart occurs through multiple pathways. Altered substrate utilization causes reduced mitochondrial oxidative metabolism. Under normal conditions the adult heart generates $70 \%$ of its ATP through fatty acid oxidation and 30\% through glucose and lactate metabolism [8]. Heart failure is not only characterized by modified ventricular mass, but also by a switch to the fetal gene expression program causing altered substrate utilization [9]. The fetal heart develops in an environment low in oxygen that preferentially uses glucose as a substrate for energy $[10,11]$. Consequently, the fetal heart has fewer mitochondria and lower citric acid cycle activity than the adult heart [12]. The heart has a plasticity to respond to a variety of stressors and can therefore modulate its sources of energy [13]. The response to cardiac stress (e.g. hypertension, pressure overload, myocardial infarction) induces cardiac remodeling processes that are accompanied by changes in gene expression patterns of fetal energy metabolism [14].

An over activated renin-angiotensin-aldosterone-system (RAAS) effects heart and kidney function [15]. Angiotensin II activates NADPH oxidase leading to an increased production of reactive oxygen species (ROS) in the heart [16] and the kidney [17]. The local RAAS in the kidney is a strong regulator of blood pressure [18]. The expression of the intrarenal angiotensin II receptors $\mathrm{AT}_{1}$ and $\mathrm{AT}_{2}$ correlate with blood pressure. Studies in rats have shown that the expression of these receptors in the kidney can be modulated in the fetus by the diet of the mother during pregnancy [19]. The expression of the $\mathrm{AT}_{1}$ receptor is increased in the fetal kidney in rats in offspring of mothers exposed to a low protein diet during pregnancy, but decreases at 12 days of age, thus showing a plasticity of expression over time after adverse insults occur [20].

Epidemiological observations in humans and experimental animal models have demonstrated that offspring exposed to adverse nutritional influences during pregnancy (in utero, IU) and/or the lactation (L) period are susceptible to metabolic and CVD in adulthood $[21,22]$. Specifically, a maternal diet low in protein during IU/L is linked to impaired nephrogenesis, reduced nephron numbers and hypertension in human [23] and rat offspring [24]. An altered prenatal environment that leads to hypertension in offspring as a result of abnormal kidney development has been linked to increases in renin gene expression [25]. Overnutrition during pregnancy is sufficient to induce cardiac hypertrophy in young offspring in mice [26].

The purpose of this study was to investigate the effect of a HFD during IU/L on the development of features of the CRS and CRMS by assessing altered cardiac morphology, gene expression and insulin responsiveness, and altered renal morphology and gene expression in adult male offspring. This study utilized the GLUT4 heterozygous (G4+/-) mouse that exhibits insulin resistance [27] and develops hypertension when exposed to a HFD during IU/L [28]. Since, to the best of our knowledge, the role of GLUT4, the major glucose transporter in the heart that is also present in the kidney [27, 28], has not been investigated in the genesis of fetal programming of CRS or CRMS, we provide a mouse model that is novel for investigating these diseases. 


\section{Kidney Blood Pressure Research}

\section{Materials and Methods}

\section{Animals and Experimental Design}

Animals were housed in a barrier facility and maintained on a 14-10 hour light-dark cycle with ad libitum access to chow and water. Age- and body weight (BW) matched WT female mice on a CD1 background were maintained on control PicoLab® Mouse Diet \#5058 (LabDiet, St. Louis, MO 63144, USA) (C: 9\% fat as soybean oil and porcine animal fat, $20 \%$ protein, $53 \%$ carbohydrate, $3.59 \mathrm{kcal} / \mathrm{g}$ ), or switched to high fat Bio-Serv Product \#F3282 (Bio-Serv, Frenchtown, NJ 08825, USA) (HFD: 35.5\% fat as lard, 20\% protein, $36.3 \%$ carbohydrate, $5.29 \mathrm{kcal} / \mathrm{g}$ ) 2 weeks prior to mating, throughout pregnancy and lactation (IU/L). The CD1 mouse model is an outbred strain and was chosen because of a strong heterogeneity in its genome [27]. WT female mice were bred to non-littermate GLUT4 heterozygote (G4+/-) males [28, 29]. Genotyping was performed as previously described [30]. WT and G4+/- male offspring from 2 - 7 litters/ group were weaned onto PicoLab® Mouse Diet \#5053 (LabDiet, St. Louis, MO 63144, USA) (low fat- LFD: 4.5\% fat as soybean oil, $20 \%$ protein, $54.8 \%$ carbohydrate, $3.4 \mathrm{kcal} / \mathrm{g}$ ) at postnatal day 21 until the age of 24 weeks. Male offspring were the focus of this study to circumvent the confounding effects of estrogen in metabolism. In addition, we previously demonstrated that only male offspring exposed to HFD during IU/L develop hypertension in adult life [28]. Body weight was recorded weekly until 12 weeks of life and then bi-weekly until the end of the study. At the end of the study, after a six hour fasting period, mice were injected intra-peritoneally (i. p.) with either short acting Humalog ${ }^{\circledR}$ insulin (Lilly, Indianapolis, IN, USA) (0.75 U/kg body weight) or $0.9 \%$ $\mathrm{NaCl} 15$ min prior to sacrificing by cervical dislocation. Hearts and kidneys were removed, weighed and placed in liquid nitrogen immediately and stored at $-80^{\circ} \mathrm{C}$ until further analysis. Heart and kidney weight was normalized to total body weight. A second cohort of pregnant mice was generated as described above for gene expression analysis in the kidney in five week old mice (animals from 2 - 3 litters/ group). Animal protocols were approved by the Animal Institute of the Albert Einstein College of Medicine.

\section{Blood Pressure Measurements}

Blood pressure measurements were performed on offspring at 7, 16 and 21 weeks of age using the CODA tail-cuff blood pressure system (Kent Scientific Corp., Torrington, CT, USA). The tail blood pressure system was used in order to perform a longitudinal blood pressure analysis in mice throughout the study. Mice were placed in a lucite holder with a built-in temperature controller and blood pressure was measured once the mice were properly warmed and tail arteries dilated. Prior to the actual blood pressure measurements mice were trained to stay in the device. Multiple measurements were performed with a minimum of 10 valid measurements for each mouse.

Quantitative Real Time-PCR Analysis

mRNA expression was performed on hearts and kidneys as previously described [31]. Primers were designed using Primer Express software (Applied Biosystems, Foster City, CA, USA). Primer sequences are listed in table 1. Data is expressed relative to the WT C group. The constitutively expressed housekeeping gene, ubiquitin, was used for data normalization.

\section{Immunoblot analysis}

Mouse heart tissue was homogenized in a buffer containing $50 \mathrm{mM}$ HEPES, 5 mM EDTA, $15 \mathrm{mM}$ Napyro-phosphate, $25 \mathrm{mM} \mathrm{NaF}, 100 \mathrm{mM} \beta$-glycerol-phosphate, $2 \mathrm{mM} \mathrm{Na}_{3} \mathrm{VO}_{4}, 10 \%$ Triton X-100, and Complete Protease Inhibitor Cocktail Tablets (Roche, Mannheim, Germany). Homogenates ( $60 \mu \mathrm{g}$ of protein) were separated by $10 \%$ SDS-PAGE and transferred to Hybond enhanced chemiluminescence (ECL) nitrocellulose (Amersham Pharmacia Biotech, Piscataway, NJ, USA). Primary antibodies for total Akt and phospho-Akt ${ }^{\text {Thr308 }}$ were used from Cell Signaling Technology (Danvers, MA, USA). Secondary anti-rabbit antibody was used from Li-Cor (Lincoln, NE, USA). Signals were visualized and quantified using the infrared Odyssey Western Blotting System (Li-Cor, Lincoln, NE, USA).

\section{Kidney morphology and nephron number measurement}

For histological analyses, kidneys were carefully harvested, total organ weight was measured and kidneys were fixed in 4\% paraformaldehyde. Kidneys were then dissected in $1 \mathrm{~mm}$ thick slices perpendicular to the longitudinal axis. Tissue sampling for morphometric and stereological analysis was performed using the area weighted sampling technique as previously described [32]. The kidney slices were embedded in 


\section{Kidney Blood Pressure Research}

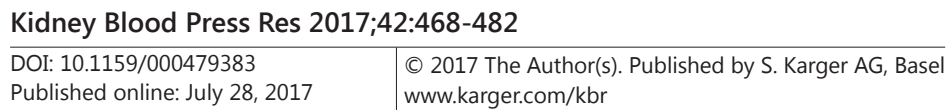

Kruse et al.: Maternal Diet and Cardio-Renal Morphology

Table 1. List of Primer Sequences. Primer sequences used for quantitative real time PCR analysis.

\begin{tabular}{llll}
\hline $\begin{array}{l}\text { Gene } \\
\text { Symbol }\end{array}$ & \multicolumn{1}{c}{ Forward Primer } & \multicolumn{1}{c}{ Reverse Primer } & \multicolumn{1}{c}{$\begin{array}{c}\text { Accession } \\
\text { Number }\end{array}$} \\
\hline Acadl & GGCTGGTTAAGTGATCTCGTGAT & ATGGGCAGGCGATCGA & NM_007381.4 \\
Acadm & AGTTTGCCAGAGAGGAGATTATCC & TGATGAGAGGGAACGGGTACTC & NM_007282.5 \\
Agtr1a & TCGCTACCTGGCCATTGTC & TGACTTTGGCCACCAGCAT & NM_177322.3 \\
Agtr1b & CCCTTCGGCAATCACCTATG & ACACACTGGCGTAGAGGTTGAA & NM_175086.3 \\
Agtr2 & CCCGTGACCAAGTCCTGAA & ATACCCATCCAGGTCAGAGCAT & NM_007429.5 \\
Col3a1 & GGGAATGGAGCAAGACAGTCTT & TGCGATATCTATGATGGGTAGTCTCA & NM_009930.2 \\
Cpt1a & AAAGATCAATCGGACCCTAGACA & CAGCGAGTAGCGCATAGTCA & NM_013495.2 \\
Cpt1b & CCAAACGTCACTGCCTAAGCT & GGCCGCACAGAATCCAAGTA & NM_009948.2 \\
Lpl & CAGCTGGGCCTAACTTTGA & AAATCAGCGTCATCAGGAGAA & NM_008509.2 \\
Nos3 & GCAATCTTCGTTCAGCCATCA & CCAGCCATGTTGGATACAGAG & NM_008713.4 \\
Nppa & GCATGGGCTCCTTCTCCAT & TGGCCTGGAAGCCAAAAG & NM_008725.3 \\
Pdk2 & AGTACATCGAGCACTTCAGCAAGT & GGATCCGAAGTCTAGAAACTGCTT & NM_133667.2 \\
Pdk4 & CCGCTTAGTGAACACTCCTTC & TCTACAAACTCTGACAGGGCTTT & NM_013743.2 \\
Plin5 & GAAATGGACCAGAGAGGTGAAGA & GGGTCCTGAGCTGTCTGATCA & NM_025874.2 \\
Ppara & TCGGCGAACTATTCGGCTG & GCACTTGTGAAAACGGCAGT & NM_011144.6 \\
Ppard & TTGAGCCCAAGTTCGAGTTTG & CGGTCTCCACACAGAATGATG & NM_011145.3 \\
Ren & GGTGCCCTCCACCAAGTG & TCAGAGGACTCATAGAGGCTGTGA & NM_031192.3 \\
Slc2a1 & GGTGTGCAGCAGCCTGTGT & CACAGTGAAGGCCGTGTTGA & NM_011400.3 \\
Slc2a4 & CTGCAAAGCGTAGGTACCAA & CCTCCCGCCCTTAGTTG & NM_009204.2 \\
Scl27a6 & GGCTTGAGGATGCCGCTTA & ACTCTGGGCTCATGCTATGAAGT & NM_001081072.1 \\
ubiquitin & GCCCAGTGTTACCACCAAGAAG & GCTCTTTTTAGATACTGTGGTGAGGAA & NM_019639.4 \\
\hline & & & \\
& & &
\end{tabular}

paraffin and $3 \mu \mathrm{m}$ thick sections were prepared and stained with hematoxylin/eosin (HE), periodic acid Schiff (PAS), and Sirius red (fibrous tissue stain). Renal morphology was investigated by light microscopy (various magnifications) as described below with the investigator being blinded to the treatment groups. Glomeruli were identified by their typical histologic appearance.

Glomerular geometry. Glomerular geometry was analyzed as described in detail previously [32, 33]: Volume density (VV) of glomeruli and cortex were measured by point counting at a magnification of 200x on HE sections and corrected for tissue shrinkage. Then, the number of glomeruli per area (NA) was assessed. The number of glomeruli per volume (NV) was calculated using the formula NV $=\mathrm{k} / \beta * \mathrm{NA1} .5 / \mathrm{VV} 0.5$ with $\mathrm{k}=1$ and $\beta=1.382$. The total number of glomeruli (Nglom) was derived from the total volume of the renal cortex (Vcortex) and the number of glomeruli per cortex volume: Nglom $=\mathrm{NV} *$ Vcortex. The mean glomerular tuft volume (vglom) was then determined according to vglom = Vglom / Nglom with Vglom being the total glomerular volume.

Analysis of glomerular capillarization and cellularity on semi-thin sections. Five semi-thin sections per animal were analyzed in terms of glomerular capillarization and cellularity using the point counting method and a 121 point eyepiece (Zeiss Co., Oberkochen, Germany) at a magnification of 1000x with oil immersion as previously described [32]. In brief, length density (LV) of glomerular capillaries was determined according to the standard stereological formula $\mathrm{LV}=2 * \mathrm{QA}$ with QA being the number of capillary transects per area of the capillary tuft. Total length of glomerular capillaries per kidney (Ltot) was then derived from LV and the total glomerular volume: Vglom $=$ VVglom $*$ Vcortex. Total volume of the capillary tuft (Vkap) was also calculated using point counting. The number of podocytes, endothelial and mesangial cells per glomerulus was assessed by stereological techniques in at least 25 glomeruli per animal from density per volume (NVcell) and volume density of the cell type (VVcell) according to NVcell $=\mathrm{k} / \beta *$ NAcell1.5 / VVcell0.5 with $\beta=1.5$ for podocytes and $\beta=1.4$ for endothelial and mesangial cells, as well as $\mathrm{k}=1$. The number of cells per glomerulus (Ncell) was determined using the formula Ncell $=$ NVcell $*$ vglom. The mean cell volume (vcell) was calculated with vcell $=$ VVcell $*$ Vglom $/$ Nglom [34] . 


\section{Kidney Blood Pressure Research}

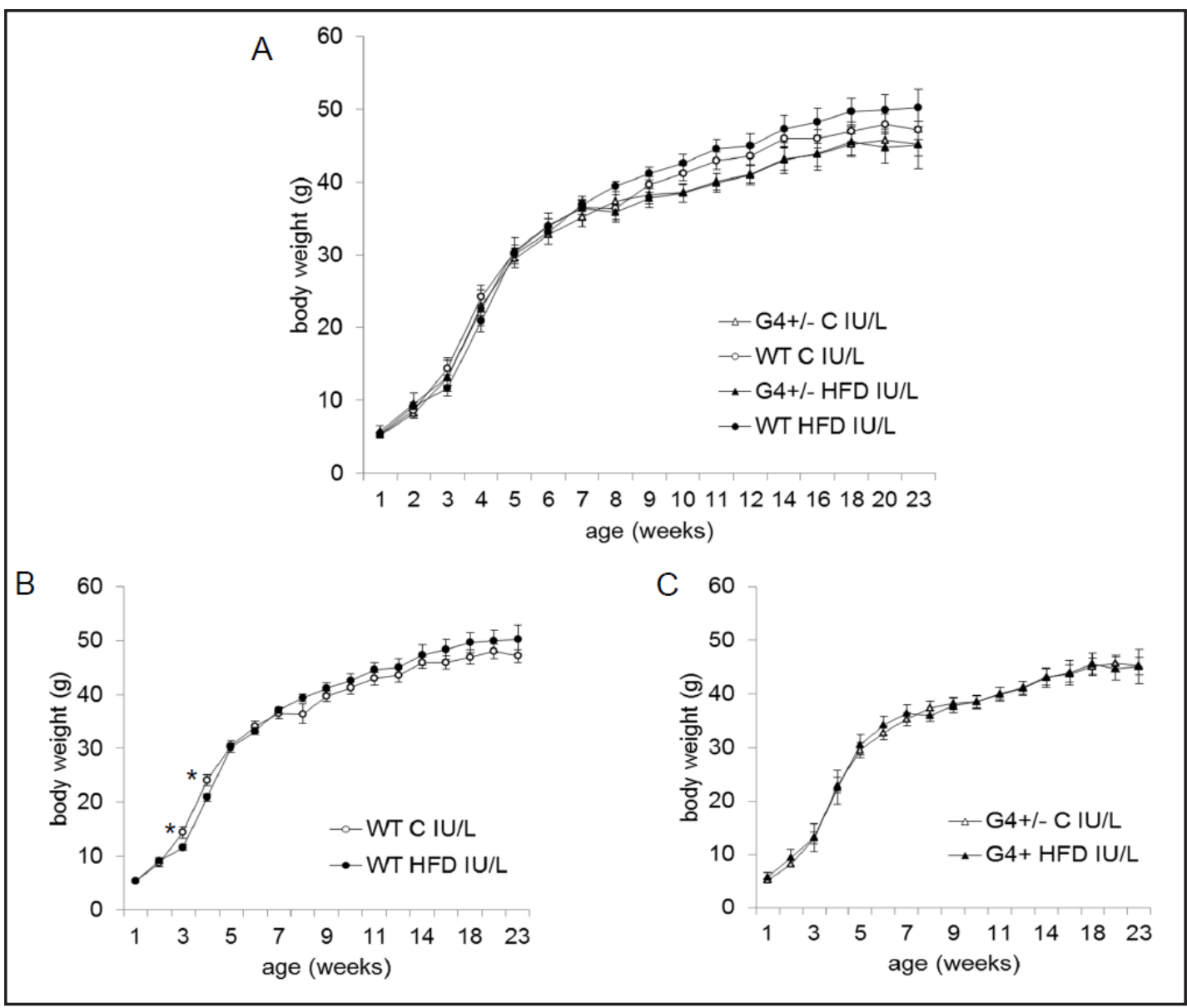

Fig. 1. Growth curves of mice. Body weights of all mice are shown in figure $1 \mathrm{~A}$. Growth curves are shown separately for WT mice (fig. 1B, ${ }^{*} \mathrm{p}<0.05, \mathrm{n}=7-9$ /group) and G4+/- mice (fig. 1C, $\mathrm{n}=4-11$ /group), respectively, on either a $\mathrm{C}$ diet or HFD during IU/L.

\section{Measurement of urinary albumin and creatinine}

Urinary albumin and creatinine were measured at 12-14 weeks from an overnight urine collection as described previously [35]. Mice were allowed to have free access to food and water. Albumin concentration was measured using the Albuwell $\mathrm{M}^{\circledast}$ mouse ELISA kit and creatinine concentration was measured using The Creatinine Companion ${ }^{\circledR}$ assay (both kits: Exocell, Philadelphia, PA, USA) according to the manufacturer's instructions.

\section{Data Analysis}

Normal distribution was tested using the Kolmogorov-Smirnov test. Statistical comparison of WT C, WT HFD, G4+/- C and G4+/- HFD was performed using two-way ANOVA analysis and one-way ANOVA analysis with Bonferroni posttest using JMP IN 5.1 software (SAS Institute, Cary, NC, USA). Additionally, for blood pressure measurements, two-way ANOVA analysis with repeated measurements was performed. Values are mean \pm SEM for each group. Significance was accepted at $\mathrm{p}<0.05$.

\section{Results}

Body, heart and kidney weight at the age of 24 weeks

Figure $1 \mathrm{~A}$ shows the BW of mice of all groups over the duration of the study. WT mice on HFD during IU/L had a significant delayed increase in BW at three and four weeks of age 


\section{Kidney Blood Pressure Research}

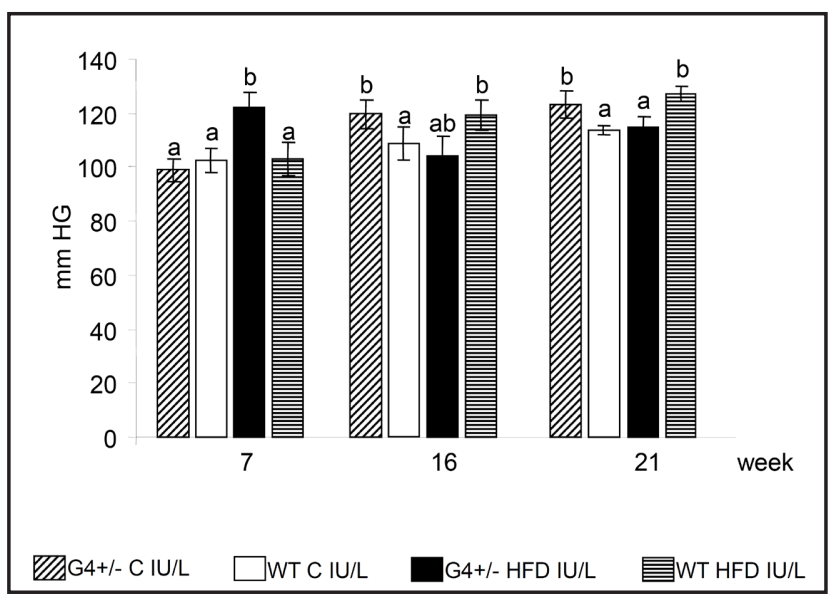

Fig. 2. Systolic blood pressure measurement. Blood pressure was measured using a tail-cuff blood pressure system at 7, 16, and 24 weeks of age. Data represent mean \pm SEM. Bars that do not share the same letter are significantly different from each other ( $<<0.05, n=5 /$ group).
Table 2. Body weight and heart and kidney weight of offspring. Heart and kidney weight at the age of 24 weeks normalized to BW (arbitrary units x 100, $\mathrm{n}=4-10$ / group) of WT or G4+/- offspring on either a $\mathrm{C}$ or HFD during IU/L and a $\mathrm{C}$ diet after weaning. Data represent mean \pm SEM, ${ }^{*} \mathrm{p}<0.05$ compared to $\mathrm{C}$ diet for the same genotype.

\begin{tabular}{lcc}
\hline & $\begin{array}{c}\text { Heart/BW } \\
(\text { wk24) }\end{array}$ & $\begin{array}{c}\text { Kidney/BW } \\
\text { (wk 24) }\end{array}$ \\
\hline G4+/- C & $0.46 \pm 0.01$ & $0.80 \pm 0.03$ \\
WT C & $0.48 \pm 0.01$ & $0.81 \pm 0.03$ \\
G4+/- HFD & $0.55 \pm 0.02^{*}$ & $0.98 \pm 0.04^{*}$ \\
WT HFD & $0.56 \pm 0.03^{*}$ & $0.96 \pm 0.03^{*}$ \\
\hline
\end{tabular}

compared to WT C IU/L (both $\mathrm{p}<0.05$ ) followed by a catch-up growth (fig. 1B). This was not seen for G4+/- HFD IU/L mice (fig. 1C). No differences in BW were observed between groups after the age of 5 weeks.

Heart and kidney weight of WT mice exposed to a HFD during IU/L was increased 1.17-fold and 1.19-fold, respectively, compared to WT mice exposed to a C diet during IU/L ( $p<0.05, n=5-10$; normalized to body weight). Heart and kidney weight of G4+/- exposed to a HFD during IU/L were increased 1.20-fold and 1.23-fold, respectively, compared to G4+/mice on a control diet during IU/L ( $\mathrm{p}<0.05, \mathrm{n}=4-10$, normalized to body weight) (table 2 ).

\section{Blood pressure measurements}

Figure 2 shows the systolic blood pressure measurement. At 7 weeks of age G4+/offspring exposed to a HFD during IU/L had a 1.19- fold increase in systolic blood pressure when compared to WT mice on a $\mathrm{C}$ diet during IU/L $(\mathrm{p}<0.05)$. WT offspring exposed to a HFD during IU/L and G4+/- mice born to mothers that consumed control diet during IU/L were normotensive at this age. At 16 weeks of age G4+/- offspring exposed to a $\mathrm{C}$ diet during IU/L and WT offspring exposed to a HFD during IU/L had a 1.10-fold and 1.09-fold increase, respectively, in blood pressure when compared to WT controls $(\mathrm{p}<0.05)$. G4+/- offspring exposed to a HFD during IU/L remained normotensive at that age. At 21 weeks of age the blood pressure pattern was similar to that measured at 16 weeks of age: G4+/- offspring exposed to a C diet during IU/L showed a 1.08-fold increase and WT offspring exposed to a HFD during IU/L showed a 1.12-fold increase in blood pressure when compared to WT controls $(\mathrm{p}<0.05)$.

\section{Cardiac mRNA expression}

Figure 3 and 4 illustrate the results of cardiac mRNA expression at 24 weeks of age. As expected, Slc2a4 expression was reduced $42 \%$ in G4+/- compared to WT on a C diet $(\mathrm{p}<0.05)$. Interestingly, HFD during IU/L did not further alter GLUT4 expression in G4+/hearts nor did it affect Slc2a4 expression in WT hearts compared to WT on C during IU/L (fig. 3A). Exposure to a HFD during IU/L resulted in a 36\% decrease in expression of the transcription factor Ppard that was significant in G4+/- offspring $(\mathrm{p}<0.05)$ but did not reach significance in WT offspring ( $21 \%$ reduction, $p=n . s$.) when compared to WT offspring on a C diet during IU/L (fig. 3B). Plin5 expression decreased 31\% in G4+/- but not in WT offspring on a HFD during IU/L ( $\mathrm{p}<0.05$, compared to WT on C diet during IU/L, fig. 3C). Cpt1a and 


\section{Kidney Blood Pressure Research}

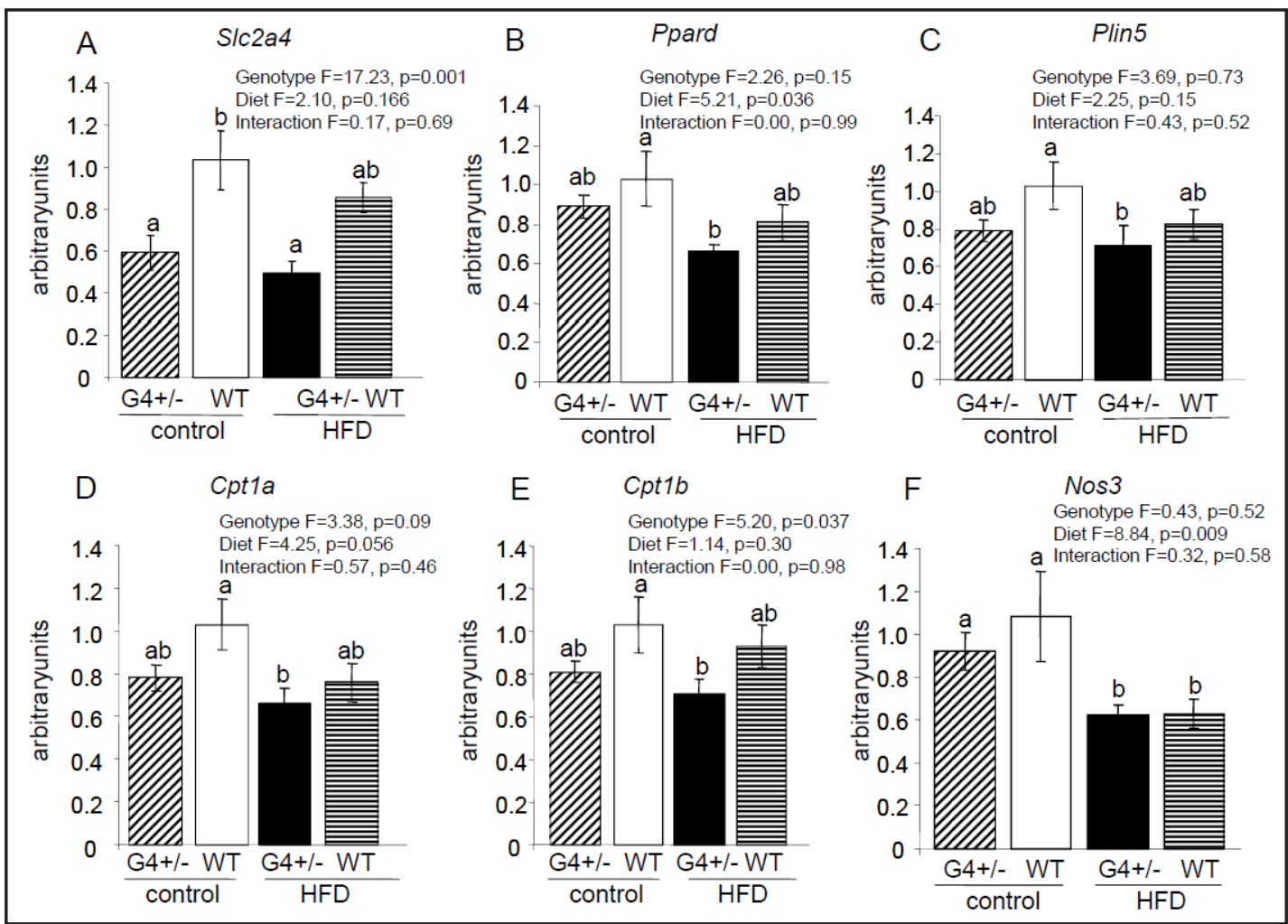

Fig. 3. Cardiac gene expression of Slc2a4 (A), PpardS (B), Plin5 (C), Cpt1a (D), Cpt1b (E) and Nos3 (F) in 24 week old male mice. Data represent mean \pm SEM. Bars that do not share the same letter are significantly different from each other ( $\mathrm{p}<0.05, \mathrm{n}=4$-6/group). 2-way ANOVA results for diet, genotype and the interaction of diet and genotype are inserted into each graph of the appropriate gene.

Cpt1b expression was decreased 31\% and 36\%, respectively, in G4+/- offspring exposed to a HFD during IU/L ( $\mathrm{p}<0.05$, compared to WT on C diet during IU/L) but not for WT offspring on a HFD during IU/L (fig. 3, D and E). HFD during IU/L resulted in a significant $41 \%$ and $42 \%$ decrease, respectively, in Nos3 expression in hearts of G4+/- and WT offspring (both $\mathrm{p}<0.05$, compared to WT on C diet during IU/L) (fig. 3F).

Interestingly, a HFD during IU/L induced a 77\% reduction of gene expression levels of fatty acid transporter 6 (Scl27a6) in WT offspring compared to G4+/- offspring at the age of 24 weeks ( $\mathrm{p}=0.034$ ) (fig. 4C). We did not observe any significant differences in gene expression levels of $L p L$ (fig. 4A), Cd36 (fig. 4B), Col3a1 (fig. 4D) or Nppa (fig 4E).

Two-way ANOVA analysis showed a significant influence of the genotype on gene expression of Slc2a4 ( $\mathrm{p}=0.001)$, Cpt1b $(\mathrm{p}=0.037)$ and Scl27a6 $(\mathrm{p}=0.034)$, a significant influence of the diet on gene expression of Ppard $(\mathrm{p}=0.036)$ and Nos3 $(\mathrm{p}=0.009)$, and a significant interaction of diet and genotype on gene expression of Scl27a6 ( $\mathrm{p}=0.006)$.

We did not observe any differences in expression of Acadl, Acadm, Pdk2, Pdk4, Ppara and Slc2a1 (data not shown) between any of the groups studied.

\section{Cardiac insulin responsiveness}

Cardiac responsiveness to the insulin signaling pathway was determined by immunoblotting for Akt-phosphorylation of hearts of 24 week old mice following an i. p. injection of either insulin or saline. As shown in figure 5, insulin stimulation of Akt phosphorylation at threonine 308 was attenuated in WT and G4+/- mice exposed to HFD during IU/L. WT on HFD during IU/L exhibited a 2.04-fold decrease in insulin stimulated Akt ${ }^{\text {(Thr308) }}$ phosphorylation compared to WT on $\mathrm{C}$ diet during IU/L $(\mathrm{p}<0.05)$. Insulin 


\section{Kidney \\ Bloód Pressure Research}

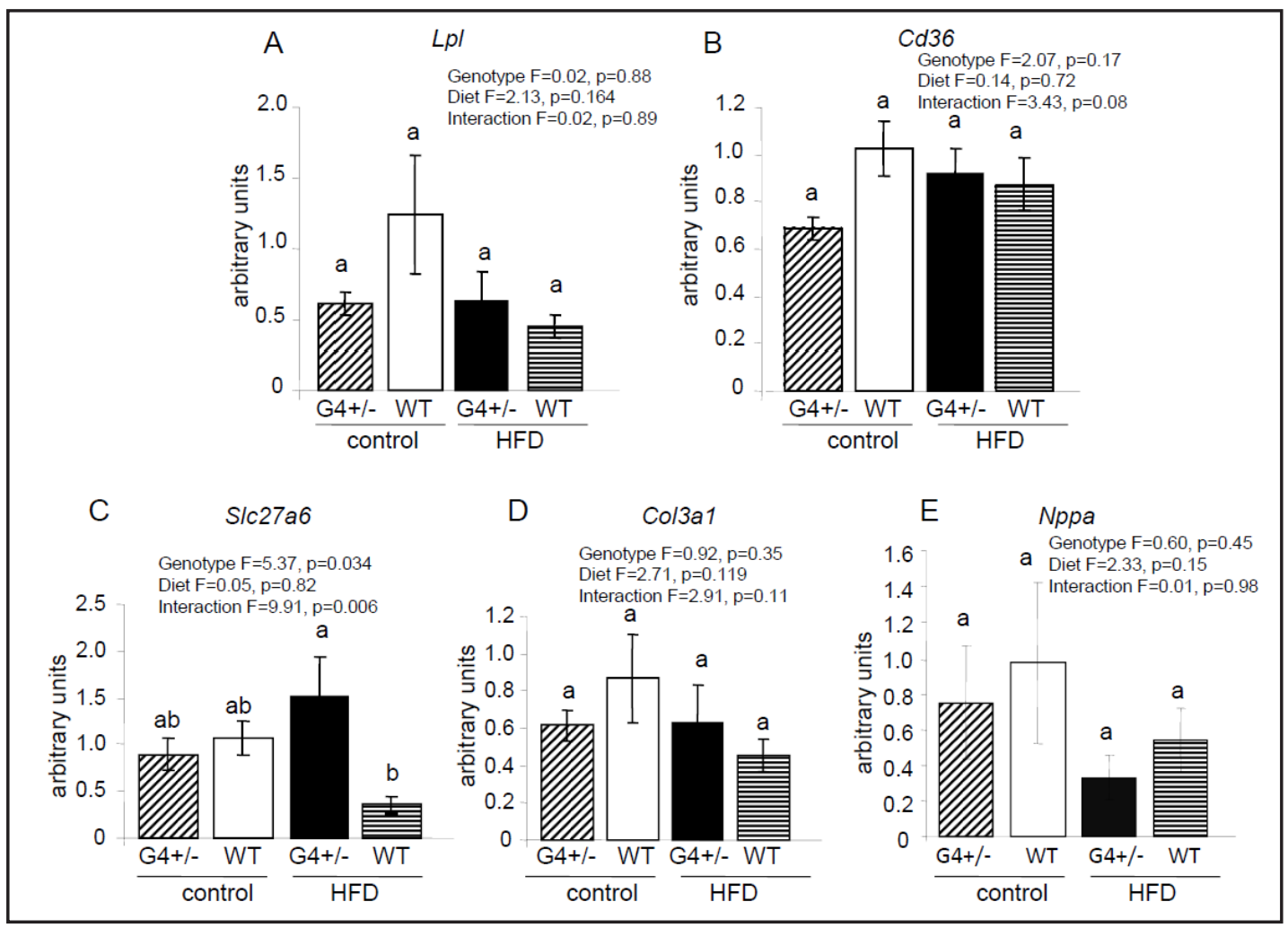

Fig. 4. Cardiac gene expression of $\mathrm{Lpl}$ (A), Cd36 (B), Slc27a6 (C), Col3a1 (D) and Nppa (E) in 24 week old male mice. Data represent mean \pm SEM. Bars that do not share the same letter are significantly different from each other ( $\mathrm{p}<0.05, \mathrm{n}=4$-6/group). 2-way ANOVA results for diet, genotype and the interaction of diet and genotype are inserted into each graph of the appropriate gene.

stimulated $\mathrm{Akt}^{(\mathrm{Thr} 308)}$ phosphorylation in G4+/- mice on HFD during IU/L was decreased 2.01-fold compared to WT on C diet during IU/L, however this did not reach significance when normalized to total Akt expression ( $\mathrm{p}=$ n.s.).

\section{Renal mRNA expression}

Since there was a strong increase of systolic blood pressure in G4+/- offspring on a HFD during IU/L at an early age, we generated a second cohort of mice to evaluate if the changes in blood pressure could be attributed to altered expression of hypertension associated genes in the kidney. At 5 weeks of age expression of the renal $\mathrm{AT}_{1} \mathrm{a}$ - and $\mathrm{AT}_{1} \mathrm{~b}$ - receptors (Agtr1a and Agtr1b) showed a 1.54-fold and 1.63-fold increase, respectively, $(\mathrm{p}<0.05)$ in G4+/- offspring on a HFD during IU/L compared to WT offspring on a C diet during IU/L (fig. 6, A and B). There was no significant difference in Agtr1a or Agtr1b expression between G4+/- offspring on a HFD during IU/L and G4+/- offspring on a C diet during IU/L or between WT offspring on a HFD during IU/L and WT offspring on a C diet during IU/L. Expression of Agtr2 was 1.85-fold increased $(\mathrm{p}<0.05)$ in G4+/- offspring on a HFD compared to G4+/- offspring on a $\mathrm{C}$ diet during IU/L (fig. 6C). WT offspring on a HFD during IU/L did not show any differences in Agtr2 expression compared to WT or G4+/- offspring on a C diet during IU/L. Ren expression in G4+/- offspring on a HFD during IU/L was increased 2.32-fold and 2.14-fold $(\mathrm{p}<0.05)$ compared to WT offspring and G4+/- offspring on a C diet during IU/L, respectively (fig. 6D). WT offspring on a HFD during IU/L exhibited a 1.95-fold increase $(\mathrm{p}<0.05)$ in Ren expression compared to WT offspring on a C diet during IU/L. Two-way ANOVA analysis showed a significant influence of the diet on gene expression of Ren $(\mathrm{p}=0.003)$. 


\section{Kidney Blood Pressure Research}

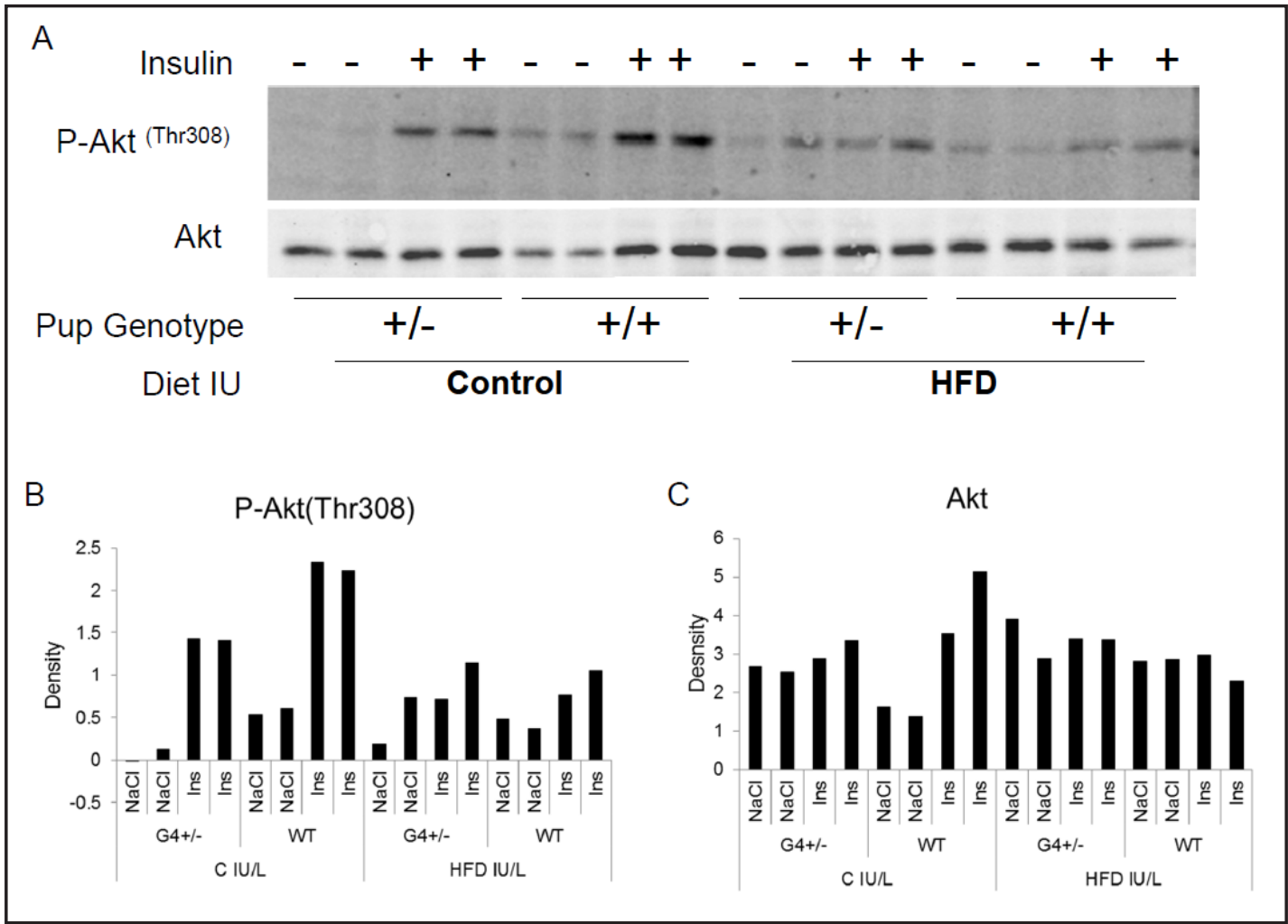

Fig. 5. Representative immunoblot for protein expression of total and phosphorylated ${ }^{(\mathrm{Thr} 308)}$ Akt in hearts at 24 weeks (fig. 1A). Immunoblots were performed on hearts of WT and G4+/- mice on either a C or HFD during IU/L sacrificed 15 minutes after an i. p. injection of $0.75 \mathrm{U} / \mathrm{kg}$ insulin or $0.9 \% \mathrm{NaCl}$. Quantitative densitometry analysis is shown for phosphorylated ${ }^{(\mathrm{Thr} 308)}$ Akt (fig. 1C) and total Akt (fig. 1B), respectively.

\section{Nephron numbers and nephron volume}

Since an altered prenatal environment could potentially change nephron numbers, we performed renal morphometric studies. G4+/- offspring on a C diet during IU/L and WT and G4+/- offspring on a HFD during IU/L showed a trend towards a reduction in nephron numbers $\left(\mathrm{N}_{\text {Glom }}\right)$, compared to WT offspring on a $\mathrm{C}$ diet during IU/L, however, this did not reach significance (table 3$)$. Similarly, mean glomerular volume $\left(\mathrm{V}_{\mathrm{Glom}}\right)$, an index of glomerular size, was not altered between the groups (table 3).

\section{Urinary albumin and creatinine concentrations}

The albumin/creatinine ratio in the urine was measured to assess renal function. This ratio did not significantly differ among the groups (table 3 ).

\section{Discussion}

Our data demonstrate that exposure to a maternal HFD during IU/L is able to induce features that characterize CRS in adult male offspring even though the mice were weaned to a low fat chow diet. It appears that the heart of 'At Risk' G4+/- offspring is more susceptible to alteration by exposure to a maternal HFD during IU/L as evidenced by a reversion to the fetal gene expression program at the age of 24 weeks. In addition, expression of genes involved in regulating blood pressure is increased in the G4+/- HFD IU/L kidney. As previously reported [27], G4+/- mice developed hypertension with aging. It is well established that dietary insults during the fetal period are able to program hypertension in adult offspring $[24,36]$. 


\section{Kidney Blood Pressure Research}

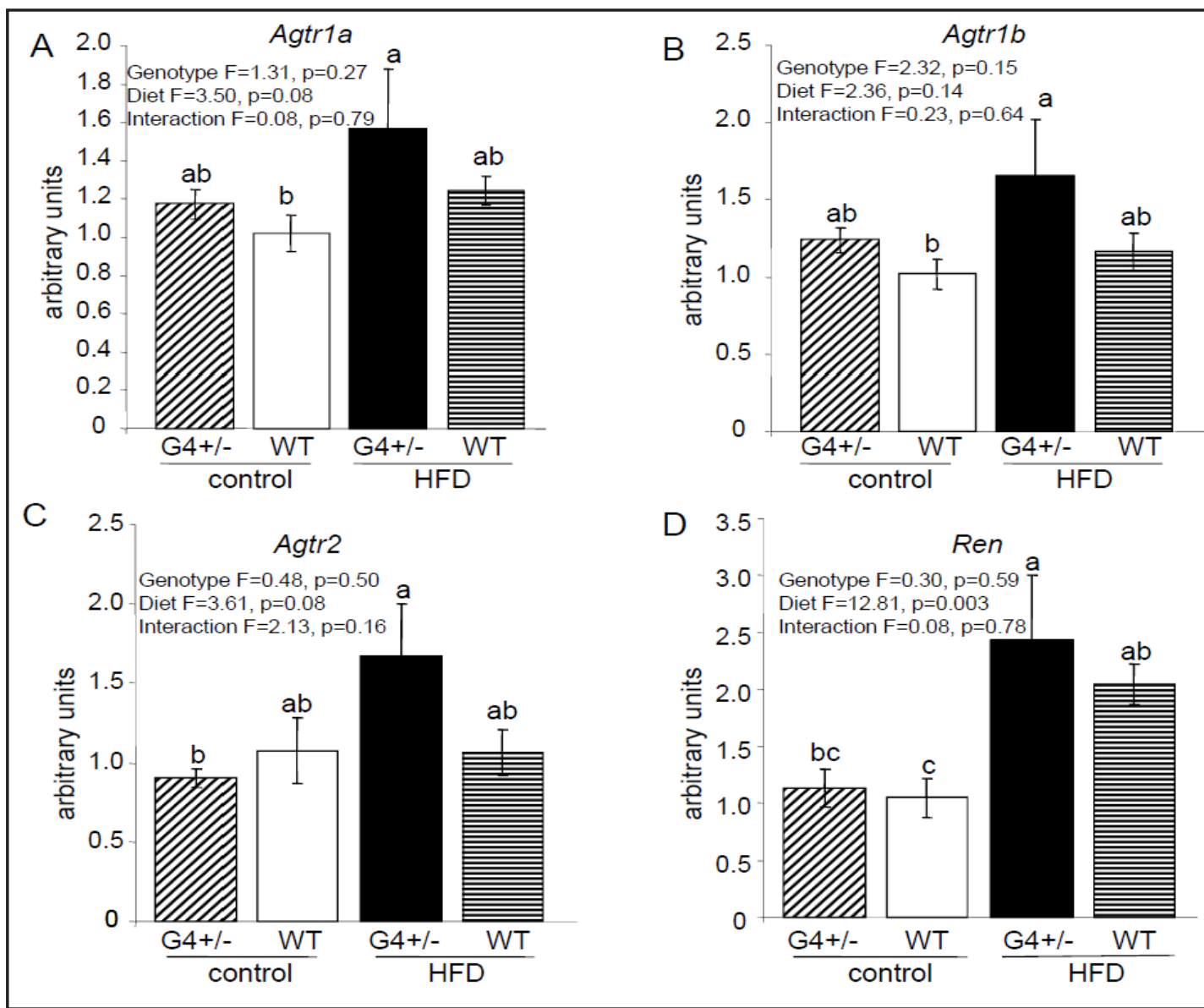

Fig. 6. Renal gene expression of Agtr1a (A), Agtr1b (B), Agtr2 (C) and Ren (D) in 5 week old male mice. Data represent mean \pm SEM. Bars that do not share the same letter are significantly different from each other ( $p<0.05, n=5$ /group). 2-way ANOVA results for diet, genotype and the interaction of diet and genotype are inserted into each graph of the appropriate gene.

Table 3. Renal morphometry and urine analysis. Renal morphometry $\left(\mathrm{N}_{\mathrm{Glom}}\right.$ : numbers of glomeruli per kidney, $\mathrm{V}_{\text {Glom }}$ : mean glomerular volume; $\mathrm{n}=4-11$ /group) of WT or G4+/offspring on either a C or HFD during IU/L and a C diet after weaning at the age of 24 weeks and urinary albumin/creatinine ratio (Alb/Crea, $n=3-4$ /group) at the age of 12-14 weeks. Data represent mean \pm SEM.

\begin{tabular}{lcccc}
\hline & G4+/- C & WT C & G4+/- HFD & WT HFD \\
\hline $\mathrm{N}_{\text {Glom }}$ & $7.718 \pm 556$ & $8.502 \pm 482$ & $7.789 \pm 845$ & $7.897 \pm 576$ \\
$\mathrm{~V}_{\text {Glom }}\left(10^{3} \mu \mathrm{m}^{3}\right)$ & $2.485 \pm 195$ & $2.350 \pm 197$ & $2.301 \pm 153$ & $2.392 \pm 270$ \\
$\mathrm{Alb} /$ Crea $(\mu \mathrm{g} / \mathrm{mg})$ (urine) & $64.4 \pm 19.2$ & $86.7 \pm 29.8$ & $103.5 \pm 40.8$ & $39.8 \pm 8.4$ \\
\hline
\end{tabular}

For offspring of G4+/- C IU/L and WT HFD IU/L blood pressure was normal at 7 weeks of age. The combination of an altered genotype (G4+/-) and HFD during IU/L however, resulted in increased systolic blood pressure at this young age. This elevated blood pressure was not further observed in older mice of this group suggesting compensatory mechanisms that normalize blood pressure. A similar result was seen in a study in rats that investigated the effect of a mismatch in pre- and post-natal diet on kidney development [37]. Specifically, offspring were exposed to a low protein diet during pregnancy, overfed by culling down the 


\section{Kidney Blood Pressure Research}

original litter size during the suckling period, and exposed to a regular diet after weaning. These mice showed reduced nephron numbers but only transient hypertension at a young age, indicating that fetal programming effects might not persist completely throughout life of an individual. Interestingly, while the hypertension in G4+/- HFD IU/L offspring did not persist, the WT offspring on HFD IU/L developed hypertension while aging. The WT HFD IU/L offspring experienced catch-up growth at a young age whereas G4+/- HFD IU/L mice did not. Indeed, catch-up growth in early life in mice has been associated with hypertension in adulthood [38].

It is known that the adult heart has the plasticity to remodel its morphometric and metabolic machinery in response to stressors such as hypertension [14, 39]. Here, we report that WT mice exposed to a HFD during IU/L display hypertension with age that is accompanied by cardiac hypertrophy. However, gene expression of markers of cardiac fibrosis (procollagen type III alpha 1, Col3a1) [40] or cardiac hypertrophy (natriuretic peptide type A, Nppa) [26] were not significantly different among the groups. At this point it is speculative whether cardiac hypertrophy could be due to left ventricular hypertrophy. Cardiac hypertrophy in these offspring may be attributed to long-standing hypertension such as that seen from 16 weeks of age in these mice. However, hypertension might not be the only explanation for cardiac hypertrophy in G4+/- offspring on a HFD during IU/L since in these mice hypertension was only observed temporarily. A more likely explanation for the cardiac abnormalities in the G4+/- offspring is the combination of fetal programming effects of a HFD during IU/L and the 'At Risk' genetic background characterized by aberrant cardiac gene expression. Interestingly, mice with a homozygote deletion of GLUT4 (G4-/-) do not show overt diabetes, but have significantly enlarged hearts without an increase in arterial blood pressure [30, 41]. The G4-/heart exhibits decreased gene expression of the rate limiting enzymes in fatty acid oxidation, Acadm and Acadl [41].

Cardiac fatty acid oxidation rapidly increases after birth, demonstrated by an increase in CPT-1 $\beta$ levels [42]. It is hypothesized that the reason for switching from the "fetal energy program" to the "adult energy program" is the change of nutrient supply after birth. The diet composition of the neonate is rich in fat and low in carbohydrates so the heart adapts to oxidizing fatty acids $[39,43]$. There is now growing evidence that the failing heart returns to the fetal program of gene expression of energy metabolism $[11,44]$. The response to cardiac stress induces cardiac remodeling processes that are accompanied by a change in expression pattern to the fetal energy metabolism program. In the failing heart, gene expression levels of transcription factors, Scl2a1, Scl2a4, Cpt1b, Acadm and Acaca are decreased [14, 39, 45]. The transcription factor Ppard, which is involved in gene expression of fatty acid oxidation, was decreased in G4+/- offspring on a HFD during IU/L. Consistent with that, Cpt1a and Cpt1b gene expression was also decreased, as seen in failing hearts [14, 39]. These data suggest the hearts of G4+/- offspring on a HFD during IU/L switch, at least partially, to a gene expression pattern seen in fetal and failing hearts whereas, WT mice on a HFD during IU/L do not. Of note, we did not see any changes in expression levels of genes involved in fatty acid uptake ( Lpl, Cd36 or Slc27a6) between G4+/- HFD IU/L and WT C IU/L. However, gene expression of Slc27a6, which encodes the fatty acid transporter 6 (FATP-6) [46], was greatly reduced in WT on a HFD IU/L when compared to G4/+ HFD IU/L. This may indicate an inability of the G4+/heart to counteract the nutritional overload of a HFD. Interestingly, HFD during IU/L resulted in a significant decrease in Nos3 gene expression, which is important for vascular relaxation [47] and was reported to be down-regulated in heart failure in mice [48].

OXPAT/PLIN5 is a member of the PAT family of proteins (perilipin, adopophilin, TIP47) that binds to lipid droplets [49]. It is highly expressed in tissues with high oxidative capacity (e. g. heart, soleus muscle, brown adipose tissue and liver), and plays a role in maintaining normal lipid metabolism [49]. Mice lacking OXPAT/PLIN5 showed increased ROS production in the heart and heart failure while aging [50]. Down-regulation of Plin5 in G4+/- offspring on a HFD during IU/L represents the deleterious programming effects in this group. The expression of relevant genes in fatty acid transport (Slc27a6) and storage (Plin5) are well studied markers of fatty acid metabolism in the heart. However, a limitation of these studies was the lack of direct measurement of lipids in the heart to more accurately reflect fatty acid metabolism. 


\section{Kidney Blood Pressure Research}

Cardiac insulin resistance is seen in CRS and CRMS, respectively [51]. This prompted us to determine whether alterations in insulin signaling (represented by Akt-phosphorylation) could be observed in hearts of 24 week old offspring exposed to HFD during IU/L. In both WT and G4+/- offspring, HFD during IU/L caused a decrease in insulin stimulated Akt ${ }^{\text {Thr308 }}$ phosphorylation. Ozanne and co-workers recently showed in WT mice that 8 week old male offspring exposed to HFD during IU/L showed cardiac hypertrophy associated with increased basal activation of the insulin signaling pathway represented by $\mathrm{Akt}^{\mathrm{Ser} 473}$-phosphorylation [26]. This observation indicates that in this setting of fetal programming the anabolic insulin pathway is pathologically activated in the development of cardiac hypertrophy in young offspring. It is conceivable that this anabolic stimulus in the heart is transiently observed early in life and may not be present in aged mice as insulin resistance worsens such as is seen in our study. Consistent with this, hearts of 24 weeks old mice that were exposed to a HFD during IU/L are unable to respond properly to insulin stimulation as evidenced by blunted Akt phosphorylation. This effect appears to be a programming effect of the diet fed during IU/L and independent of genotype.

An activated intra-renal RAAS [52] and reduced nephron numbers [53] have been shown to induce hypertension. Moreover, the RAAS and nephron quantity in offspring were altered upon different insults during IU/L. Undernutrition during IU/L leads to reduced nephron numbers in the kidney in offspring [54]. Protein restriction during IU/L in a rat model induced increased gene expression of the renal $\mathrm{AT}_{1} \mathrm{a}-$ and $\mathrm{AT}_{2}$ - receptor, but not of the renal $\mathrm{AT}_{1} \mathrm{~b}$-receptor, in offspring at postnatal day 28 [55]. In contrast, the effect of a HFD during IU/L on kidney development and the renal RAAS is not clear. Interestingly, it was shown that offspring of mice exposed to HFD during IU/L had higher kidney volume and increased nephron numbers at embryonic day 18.5 [56]. However, no long-term renal health problems were observed in this study. A study in rats showed increased blood pressure but no differences in kidney weight, glomerular number or volume and reduced renin activity in 180 day old offspring on a HFD during IU/L and a C diet after weaning [57]. In contrast, our mouse study revealed an increased kidney/body weight ratio in 24 week old WT and G4+/offspring on a HFD during IU/L. We did not observe any alterations in nephron number or glomerular volume. Increased gene expression of Agtr1a, Agtr1b and Agtr2 was seen only in kidneys of young G4+/- offspring when catch-up growth was completed (5 weeks of age in a duplicate cohort) on a HFD during IU/L. These results are in agreement with the precocious manifestation of hypertension in young G4+/-HFD IU/L offspring (7 weeks of age) in the first cohort. Interestingly, another study using mice showed that the presence of intra-renal $\mathrm{AT}_{1}$-receptors is essential for developing hypertension and cardiac hypertrophy [58].

A limitation of the study is the use of the tail-cuff blood pressure system since animals needed to be measured multiple times during the study. Studies using radiotelemetry for blood pressure recording are needed in the future to confirm these data. Another limitation is the use of animals from 2-7 litters/group and 2-3 litters/group, respectively. However, the CD1 mice model is an outbred strain ensuring strong inter-individual genetic heterogeneity.

\section{Conclusion}

We have demonstrated that a HFD during IU/L programs cardiac and renal alterations associated with CRS in offspring. It appears that exposure to HFD during IU/L alters gross morphology of the hearts and heart failure up to 24 weeks of life irrespective of the genotypes investigated. Hearts of both HFD IU/L genotypes were enlarged and showed decreased Nos3 gene expression. However, cardiac metabolism of G4+/- mice seems to be more susceptible to the nutritional overload of a HFD during pregnancy as demonstrated by alterations in cardiac gene expression. Although only a mild renal phenotype of kidney enlargement was observed, early alterations of the intra-renal RAAS are supportive of early hypertension in G4+/- mice. Our observations are in line with previous studies indicating that fetal programming effects 


\section{Kidney Bloód Pressure Research}

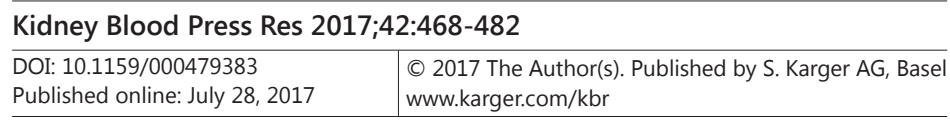

Kruse et al.: Maternal Diet and Cardio-Renal Morphology

are not consistently present and might vary during development of offspring. More studies are needed, specifically in the G4+/- genotype, to reveal the long-term effects of the maternal HFD on blood pressure and cardiac and renal morphology.

\section{Disclosure statement}

No conflicts of interest declared.

\section{Acknowledgements}

We would like to thank Kirsten Hartil, Ph. D. for the support in maintaining the mouse strain. We appreciate the excellent technical assistance of Carolyn Hung, Miriam Reutelshoefer, Katrin Schmitt and Monika Klewer.

This work was supported by National Institutes of Health (R21 DK081194 to MJC, Diabetes Research and Training Center P60 DK020541, Epigenomics, Liver, O’Brien Kidney, and Comprehensive Cancer Centers of Albert Einstein College of Medicine), Diabetes Action Foundation (to MJC and MK) and American Diabetes Association (to MJC). JT has received grant support from the National Natural Sciences Foundation of China (ref. no. 81170665).

\section{References}

1 Goh CY, Vizzi G, De Cal M, Ronco C: Cardiorenal syndrome: a complex series of combined heart/kidney disorders. Contrib Nephrol 2011;174:33-45.

-2 Virzì GM, Clementi A, Brocca A, de Cal M, Ronco C: Molecular and Genetic Mechanisms Involved in the Pathogenesis of Cardiorenal Cross Talk. Pathobiology 2016;83:201-210.

3 Ronco C, Haapio M, House AA, Anavekar N, Bellomo R: Cardiorenal syndrome: J Am Coll Cardiol 2008;52:15271539.

4 Rubattu S, Mennuni S, Testa M, Mennuni M, Pierelli G, Pagliaro B, Gabriele E, Coluccia R, Autore C, Volpe M: Pathogenesis of chronic cardiorenal syndrome: is there a role for oxidative stress? Int J Mol Sci 2013;14:23011-23032.

5 Whaley-Connell A, Sowers JR: Basic science: Pathophysiology: the cardiorenal metabolic syndrome. J Am Soc Hypertens 2014;8:604-606.

6 Ford ES: Risks for all-cause mortality, cardiovascular disease, and diabetes associated with the metabolic syndrome: a summary of the evidence. Diabetes Care 2005;28:1769-1778.

7 Avelar E, Cloward TV, Walker JM, Farney RJ, Strong M, Pendleton RC, Segerson N, Adams TD, Gress RE, Hunt SC, Litwin SE: Left ventricular hypertrophy in severe obesity: interactions among blood pressure, nocturnal hypoxemia, and body mass. Hypertension 2007;49:34-39.

8 An D, Rodrigues B: Role of changes in cardiac metabolism in development of diabetic cardiomyopathy. Am J Physiol Heart Circ Physiol 2006;291:H1489-1506.

9 Kuwahara K, Nishikimi T, Nakao K: Transcriptional regulation of the fetal cardiac gene program. J Pharmacol Sci 2012;119:198-203.

10 Battaglia FC, Meschia G: Principal substrates of fetal metabolism. Physiol Rev 1978;58:499-527.

11 Lopaschuk GD, Spafford MA, Marsh DR: Glycolysis is predominant source of myocardial ATP production immediately after birth. Am J Physiol 1991;261:H1698-1705.

$>12$ Goodwin CW, Mela L, Deutsch C, Forster RE, Miller LD, Kelivoria-Papadopoulos M: Development and adaptation of heart mitochondrial respiratory chain function in fetus and in newborn. Adv Exp Med Biol 1976;75:713-719.

13 Ouwens DM, Boer C, Fodor M, de Galan P, Heine RJ, Maassen JA, Diamant M: Cardiac dysfunction induced by high-fat diet is associated with altered myocardial insulin signalling in rats. Diabetologia 2005;48:12291237. 


\section{Kidney \\ Blood Pressure Research}

Kruse et al.: Maternal Diet and Cardio-Renal Morphology

14 Razeghi P, Young ME, Alcorn JL, Moravec CS, Frazier OH, Taegtmeyer H: Metabolic gene expression in fetal and failing human heart. Circulation 2001;104:2923-2931.

15 Giam B, Kaye DM, Rajapakse NW: Role of Renal Oxidative Stress in the Pathogenesis of the Cardiorenal Syndrome. Heart Lung Circ 2016;25:874-880.

-16 Kimura S, Zhang GX, Nishiyama A, Shokoji T, Yao L, Fan YY, Rahman M, Suzuki T, Maeta H, Abe Y: Role of NAD (P) H oxidase-and mitochondria-derived reactive oxygen species in cardioprotection of ischemic reperfusion injury by angiotensin II. Hypertension 2005;45:860-866.

17 Lodha S, Dani D, Mehta R, Bhaskaran M, Reddy K, Ding G, Singhal PC: Angiotensin II-induced mesangial cell apoptosis: Role of oxidative stress. Mol Med 2002;8:830-840.

18 Lu X, Roksnoer LC, Danser AH: The intrarenal renin-angiotensin system: does it exist? Implications from a recent study in renal angiotensin-converting enzyme knockout mice. Nephrol Dial Transplant 2013;28:2977-2982.

19 Sahajpal V, Ashton N: Increased glomerular angiotensin II binding in rats exposed to a maternal low protein diet in utero. J Physiol 2005;563:193-201.

20 Mesquita FF, Gontijo JA, Boer PA: Maternal undernutrition and the offspring kidney: from fetal to adult life. Braz J Med Biol Res 2010;43:1010-1018.

-21 Langley-Evans SC, McMullen S: Developmental origins of adult disease. Med Princ Pract 2010;19:87-98.

22 Williams L, Seki Y, Vuguin PM, Charron MJ: Animal models of in utero exposure to a high fat diet: A review. Biochim Biophys Acta 2014;1842:507-519.

23 Benz K, Amann K: Maternal nutrition, low nephron number and arterial hypertension in later life. Biochim Biophys Acta 2010;1802:1309-1317.

24 Langley-Evans SC, Welham SJ, Jackson AA: Fetal exposure to a maternal low protein diet impairs nephrogenesis and promotes hypertension in the rat. Life Sci 1999;64:965-974.

25 Kingdom JC, Hayes M, McQueen J, Howatson AG, Lindop GB: Intrauterine growth restriction is associated with persistent juxtamedullary expression of renin in the fetal kidney. Kidney Int 1999;55:424-429.

-26 Fernandez-Twinn DS, Blackmore HL, Siggens L, Giussani DA, Cross CM, Foo R, Ozanne SE. The programming of cardiac hypertrophy in the offspring by maternal obesity is associated with hyperinsulinemia, AKT, ERK, and mTOR activation. Endocrinology 2012;153:5961-5971.

27 Stenbit AE, Tsao TS, Li J, Burcelin R, Geenen DL, Factor SM, Houseknecht K, Katz EB, Charron MJ: GLUT4 heterozygous knockout mice develop muscle insulin resistance and diabetes. Nat Med 1997;3:1096-1101.

28 Vuguin PM, Hartil K, Kruse M, Kaur H, Lin CL, Fiallo A, Glenn AS, Patel A, Williams L, Seki Y, Katz EB, Charron MJ: Shared effects of genetic and intrauterine and perinatal environment on the development of metabolic syndrome. PLoS One 2013;8:e63021.

29 Hartil K, Vuguin PM, Kruse M, Schmuel E, Fiallo A, Vargas C, Warner MJ, Durand JL, Jelicks LA, Charron MJ: Maternal substrate utilization programs the development of the metabolic syndrome in male mice exposed to high fat in utero. Pediatr Res 2009;66:368-373.

30 Katz EB, Stenbit AE, Hatton K, DePinho R, Charron MJ: Cardiac and adipose tissue abnormalities but not diabetes in mice deficient in GLUT4. Nature 1995;377:151-155.

-31 Ranalletta M, Du XQ, Seki Y, Glenn AS, Kruse M, Fiallo A, Estrada I, Tsao TS, Stenbit AE, Katz EB, Charron MJ: Hepatic response to restoration of GLUT4 in skeletal muscle of GLUT4 null mice. Am J Physiol Endocrinol Metab 2007;293:E1178-1187.

-32 Amann K, Nichols C, Tornig J, Schwarz U, Zeier M, Mall G, Ritz E: Effect of ramipril, nifedipine, and moxonidine on glomerular morphology and podocyte structure in experimental renal failure. Nephrol Dial Transplant 1996;11:1003-1011.

33 Weibel ER: Morphometry of the human lung: the state of the art after two decades. Bull Eur Physiopathol Respir 1979;15:999-1013.

-34 Haas CS, Amann K, Schittny J, Blaser B, Muller U, Hartner A: Glomerular and renal vascular structural changes in alpha8 integrin-deficient mice. J Am Soc Nephrol 2003;14:2288-2296.

-35 Roeck F, Hadeler KP, Rammensee HG, Overath P: Quantitative analysis of mouse urine volatiles: in search of MHC-dependent differences. PLoS One 2007;2:e429.

-36 Samuelsson AM, Matthews PA, Argenton M, Christie MR, McConnell JM, Jansen EH, Piersma AH, Ozanne SE, Twinn DF, Remacle C, Rowlerson A, Poston L, Taylor PD: Diet-induced obesity in female mice leads to offspring hyperphagia, adiposity, hypertension, and insulin resistance: a novel murine model of developmental programming. Hypertension 2008;51:383-392. 


\section{Kidney \\ Blood Pressure Research}

37 Boubred F, Daniel L, Buffat C, Feuerstein JM, Tsimaratos M, Oliver C, Dignat-George F, Lelièvre-Pégorier M, Simeoni U: Early postnatal overfeeding induces early chronic renal dysfunction in adult male rats. Am J Physiol Renal Physiol 2009;297:F943-951.

-38 Bol V, Desjardins F, Reusens B, Balligand J-L, Remacle C: Does Early Mismatched Nutrition Predispose to Hypertension and Atherosclerosis, in Male Mice? PLoS One 2010;5:e12656.

-39 Rajabi M, Kassiotis C, Razeghi P, Taegtmeyer H: Return to the fetal gene program protects the stressed heart: a strong hypothesis. Heart Fail Rev 2007;12:331-343.

40 Zhong J, Basu R, Guo D, Chow FL, Byrns S, Schuster M, Loibner H, Wang XH, Penninger JM, Kassiri Z, Oudit GY: Angiotensin-converting enzyme 2 suppresses pathological hypertrophy, myocardial fibrosis, and cardiac dysfunction. Circulation 2010;122:717-728.

-41 Stenbit AE, Katz EB, Chatham JC, Geenen DL, Factor SM, Weiss RG, Tsao TS, Malhotra A, Chacko VP, Ocampo C, Jelicks LA, Charron MJ: Preservation of glucose metabolism in hypertrophic GLUT4-null hearts. Am J Physiol Heart Circ Physiol 2000;279:H313-318.

-42 Onay-Besikci A, Campbell FM, Hopkins TA, Dyck JR, Lopaschuk GD: Relative importance of malonyl CoA and carnitine in maturation of fatty acid oxidation in newborn rabbit heart. Am J Physiol Heart Circ Physiol 2003;284:H283-289.

43 Girard J, Ferre P, Pegorier JP, Duee PH: Adaptations of glucose and fatty acid metabolism during perinatal period and suckling-weaning transition. Physiol Rev 1992;72:507-562.

$\checkmark 44$ Taegtmeyer H, Sen S, Vela D: Return to the fetal gene program: a suggested metabolic link to gene expression in the heart. Ann N Y Acad Sci 2010;1188:191-198.

45 Sack MN, Disch DL, Rockman HA, Kelly DP: A role for Sp and nuclear receptor transcription factors in a cardiac hypertrophic growth program. Proc Natl Acad Sci USA 1997;94:6438-6443.

46 Gimeno RE, Ortegon AM, Patel S, Punreddy S, Ge P, Sun Y, Lodish HF, Stahl A: Characterization of a heartspecific fatty acid transport protein. J Biol Chem 2003;278:16039-16044.

-47 Fleming I, Busse R: Molecular mechanisms involved in the regulation of the endothelial nitric oxide synthase. Am J Physiol Regul Integr Comp Physiol 2003;284:R1-12.

-48 Feng Q Song W, Lu X, Hamilton JA, Lei M, Peng T, Yee SP: Development of heart failure and congenital septal defects in mice lacking endothelial nitric oxide synthase. Circulation 2002;106:873-879.

49 Wolins NE, Quaynor BK, Skinner JR, Tzekov A, Croce MA, Gropler MC, Varma V, Yao-Borengasser A, Rasouli N, Kern PA, Finck BN, Bickel PE. OXPAT/PAT-1 is a PPAR-induced lipid droplet protein that promotes fatty acid utilization. Diabetes 2006;55:3418-3428.

50 Kuramoto K, Okamura T, Yamaguchi T, Nakamura TY, Wakabayashi S, Morinaga H, Nomura M, Yanase T, Otsu K, Usuda N, Matsumura S, Inoue K, Fushiki T, Kojima Y, Hashimoto T, Sakai F, Hirose F, Osumi T: Perilipin 5, a lipid droplet-binding protein, protects heart from oxidative burden by sequestering fatty acid from excessive oxidation. J Biol Chem 2012;287:23852-23863.

51 Pulakat L, Aroor AR, Gul R, Sowers JR: Cardiac insulin resistance and microRNA modulators. Exp Diabetes Res 2012;2012:654904.

-52 Kobori H, Nangaku M, Navar LG, Nishiyama A: The intrarenal renin-angiotensin system: from physiology to the pathobiology of hypertension and kidney disease. Pharmacol Rev 2007;59:251-287.

53 Keller G, Zimmer G, Mall G, Ritz E, Amann K: Nephron number in patients with primary hypertension. N Engl J Med 2003;348:101-108.

54 Vaccari B, Mesquita FF, Gontijo JA, Boer PA: Fetal kidney programming by severe food restriction: Effects on structure, hormonal receptor expression and urinary sodium excretion in rats. J Renin Angiotensin Aldosterone Syst 2015;16:33-46.

55 Vehaskari VM, Stewart T, Lafont D, Soyez C, Seth D, Manning J: Kidney angiotensin and angiotensin receptor expression in prenatally programmed hypertension. Am J Physiol Renal Physiol 2004;287:F262-267.

56 Hokke S, Puelles VG, Armitage JA, Fong K, Bertram JF, Cullen-McEwen LA: Maternal Fat Feeding Augments Offspring Nephron Endowment in Mice. PLoS One 2016;11:e0161578.

-57 Armitage JA, Lakasing L, Taylor PD, Balachandran AA, Jensen RI, Dekou V, Ashton N, Nyengaard JR, Poston L: Developmental programming of aortic and renal structure in offspring of rats fed fat-rich diets in pregnancy. J Physiol 2005;565:171-184.

58 Crowley SD, Gurley SB, Herrera MJ, Ruiz P, Griffiths R, Kumar AP, Kim HS, Smithies O, Le TH, Coffman TM: Angiotensin II causes hypertension and cardiac hypertrophy through its receptors in the kidney. Proc Natl Acad Sci USA 2006;103:17985-17990. 